\title{
Micro-earthquake monitoring with sparsely sampled data
}

\author{
Paul Sava
}

Received: 1 October 2010/Accepted: 10 February 2011/Published online: 3 March 2011

(C) The Author(s) 2011. This article is published with open access at Springerlink.com

\begin{abstract}
Micro-seismicity can be used to monitor the migration of fluids during reservoir production and hydrofracturing operations in brittle formations or for studies of naturally occurring earthquakes in fault zones. Microearthquake locations can be inferred using wave-equation imaging under the exploding reflector model, assuming densely sampled data and known velocity. Seismicity is usually monitored with sparse networks of seismic sensors, for example located in boreholes. The sparsity of the sensor network itself degrades the accuracy of the estimated locations, even when the velocity model is accurately known. This constraint limits the resolution at which fluid pathways can be inferred. Wavefields reconstructed in known velocity using data recorded with sparse arrays can be described as having a random character due to the incomplete interference of wave components. Similarly, wavefields reconstructed in unknown velocity using data recorded with dense arrays can be described as having a random character due to the inconsistent interference of wave components. In both cases, the random fluctuations obstruct focusing that occurs at source locations. This situation can be improved using interferometry in the imaging process. Reverse-time imaging with an interferometric imaging condition attenuates random fluctuations, thus producing crisper images which support the process of robust automatic micro-earthquake location. The similarity of random wavefield fluctuations due to model fluctuations and sparse acquisition is illustrated in this paper with a realistic synthetic example.
\end{abstract}

Keywords Microearthquakes · Imaging · Interferometry

P. Sava $(\square)$

Center for Wave Phenomena, Colorado School of Mines,

1500 Illinois Str., Golden, CO 80401, USA

e-mail: psava@mines.edu

\section{Introduction}

Seismic imaging based on the single scattering assumption, also known as Born approximation, consists of two main steps: wavefield reconstruction which serves the purpose of propagating recorded data from the acquisition surface back into the subsurface, followed by an imaging condition which serves the purpose of highlighting locations where scattering occurs.

This framework holds both when the source of seismic waves is located in the subsurface and the imaging target consists of locating this source, as well as when the source of seismic waves is located on the acquisition surface and the imaging target consists of locating the places in the subsurface where scattering or reflection occurs. In this paper, I concentrate on the case of imaging seismic sources located in the subsurface, although the methodology discussed here applies equally well for the more conventional imaging with artificial sources.

An example of seismic source located in the subsurface is represented by micro-earthquakes triggered by natural causes or by fluid injection during reservoir production or fracturing. One application of micro-earthquake location is monitoring of fluid injection in brittle reservoirs when micro-earthquake evolution in time correlates with fluid movement in reservoir formations. Micro-earthquakes can be located using several methods including double-difference algorithms (Waldhauser and Ellsworth 2000), Gaussian-beam migration (Rentsch et al. 2004, 2007), diffraction stacking (Gajewski et al. 2007) or time-reverse imaging (Gajewski and Tessmer 2005; Artman et al. 2010).

Micro-earthquake location using time-reverse imaging, which is also the technique advocated in this paper, follows the same general pattern mentioned in the preceding paragraph: wavefield-reconstruction backward in time 


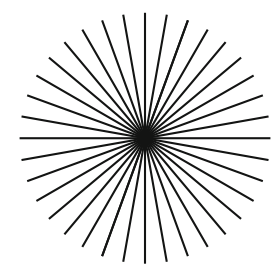

(a)

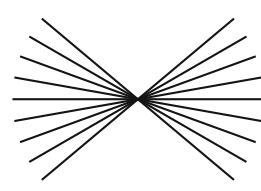

(b)

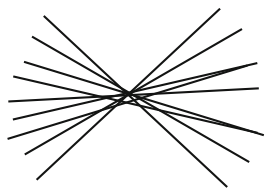

(c)

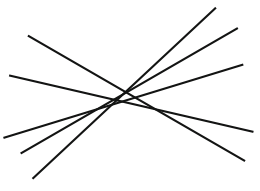

(d)
Fig. 1 Schematic representation of focus constructions using time reversal. Each line in the plots represents a wavefront reconstructed at the source from a given receiver. The panels represent the following cases: a dense acquisition, complete angular coverage and correct velocity, b dense acquisition, partial angular coverage and correct

followed by an imaging condition extracting the image, i.e. the location of the source. The main difficulty with this procedure is that the onset of the micro-earthquake is unknown, i.e. time $t=0$ is unknown, so the imaging condition cannot be simply applied as it is usually done in zero-offset migration. Instead, an automatic search needs to be performed in the back-propagated wavefield to identify the locations where wavefield energy focuses. This process is difficult and often ambiguous since false focusing locations might overlap with locations of wavefield focusing. This is particularly true when imaging using an approximate model which does not explain all random fluctuations observed in the recorded data. This problem is further complicated if the acquisition array is sparse, e.g. when receivers are located in a borehole. In this case, the sparsity of the array itself leads to artifacts in the reconstructed wavefield which makes the automatic picking of focused events even harder.

The process by which sampling artifacts are generated is explained in Fig. 1a-d. Each segment in Fig. 1a corresponds to a wavefront reconstructed from a receiver. For dense, uniform and wide-aperture receiver coverage and for reconstruction using accurate velocity, the wavefronts overlap at the source position (Fig. 1b). This idealized situation resembles the coverage typical for medical imaging, although the physical processes used are different. However, if the velocity used for wavefield reconstruction is inaccurate, then the wavefronts do not all overlap at the source position (Fig. 1c), thus leading to imaging artifacts. Likewise, if receiver sampling is sparse, reconstruction at the source position is incomplete (Fig. 1d), even if the velocity used for reconstruction is accurate. The cartoons depicted in Fig. 1a-d represent an ideal situation with receivers surrounding the seismic source, which is not typical for seismic experiments. In those cases, source illumination is limited to a range which correlates with the receiver coordinates.

In general, artifacts caused by unknown velocity fluctuations and receiver sampling overlap and, although the two phenomena are not equivalent, their effect on the velocity, c dense acquisition, partial angular coverage and incorrect velocity, and d sparse acquisition, partial angular coverage and incorrect velocity. Panel d represents the worst case scenario for micro-earthquake imaging

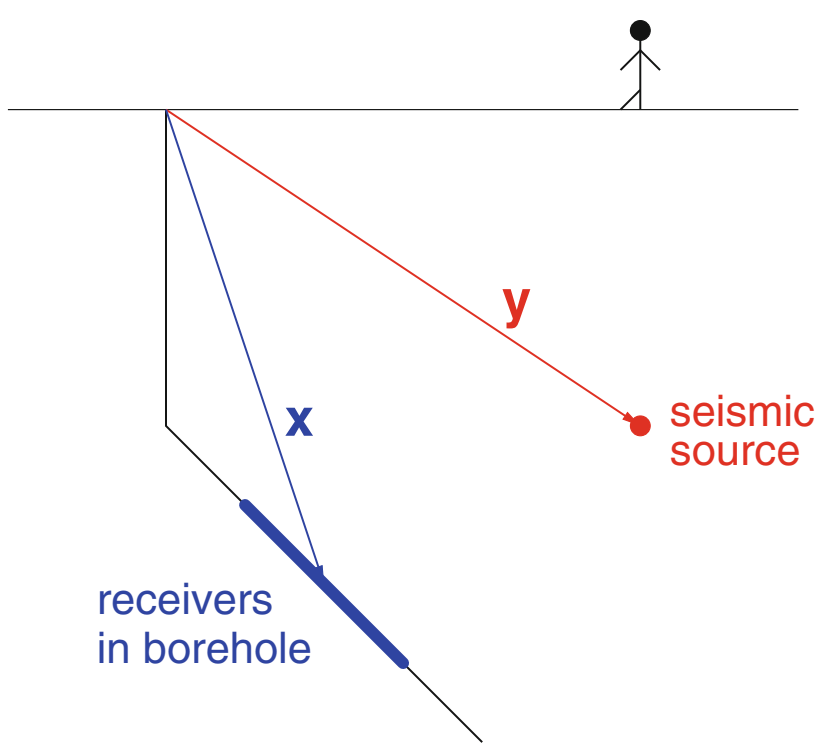

Fig. 2 Illustration of the variables $\mathbf{x}$ and $\mathbf{y}$ used for the description of the conventional and interferometric imaging procedures

reconstructed wavefields are analogous. As illustrated in the following sections, the general character of those artifacts is that of random wavefield fluctuations. Ideally, the imaging procedure should attenuate those random wavefield fluctuations irrespective of their cause in order to support automatic source identification.

\section{Conventional imaging condition}

Assuming data $D(\mathbf{x}, t)$ acquired at coordinates $\mathbf{x}$ function of time $t$ (e.g. in a borehole) we can reconstruct the wavefield $V(\mathbf{x}, \mathbf{y}, t)$ at coordinates $\mathbf{y}$ in the imaging volume using an appropriate Green's function $G(\mathbf{x}, \mathbf{y}, t)$ corresponding to the locations $\mathbf{x}$ and $\mathbf{y}$ (Fig. 2)

$V(\mathbf{x}, \mathbf{y}, t)=D(\mathbf{x}, t) *_{t} G(\mathbf{x}, \mathbf{y}, t)$,

where the symbol ${ }_{t}$ indicates time convolution. The total wavefield $U(\mathbf{y}, t)$ at coordinates $\mathbf{y}$ due to data recorded at 
all receivers located at coordinates $\mathbf{x}$ is represented by the superposition of the reconstructed wavefields $V(\mathbf{x}, \mathbf{y}, t)$ :

$U(\mathbf{y}, t)=\int_{\mathbf{x}} \mathrm{d} \mathbf{x} V(\mathbf{x}, \mathbf{y}, t)$.

A conventional imaging condition (CIC) applied to this reconstructed wavefield extracts the image $R_{\mathrm{CIC}}(\mathbf{y})$ as the wavefield at time $t=0$

$R_{\mathrm{CIC}}(\mathbf{y})=U(\mathbf{y}, t=0)$.

This imaging procedure succeeds if several assumptions are fulfilled: first, the velocity model used for imaging has to be accurate; second, the numeric solution to the waveequation used for wavefield reconstruction has to be accurate; third, the data need to be sampled densely and uniformly on the acquisition surface. In this paper, I assume that the first and third assumptions are not fulfilled. In these cases, the imaging is not accurate because contributions to the reconstructed wavefield from the receiver coordinates do not interfere constructively, thus leading to imaging artifacts. As indicated earlier, this situation is analogous to the case of imaging with an inaccurate velocity model, e.g. imaging with a smooth velocity of data corresponding to geology characterized by rapid velocity variations.

Different image processing procedures can be employed to reduce the random wavefield fluctuations. The procedure advocated in this paper uses interferometry for noise cancellation. Interferometric procedures can be formulated in various frameworks, e.g. coherent interferometric imaging (Borcea et al. 2006) or wave-equation migration with an interferometric imaging condition (Sava and Poliannikov 2008).

\section{Interferometric imaging condition}

Migration with an interferometric imaging condition (IIC) uses the same generic framework as the one used for the conventional imaging condition, i.e. wavefield reconstruction followed by an imaging condition. However, the difference is that the imaging condition is not applied to the reconstructed wavefield directly, but it is applied to the wavefield which has been transformed using pseudo-Wigner distribution functions (WDF) (Wigner 1932). By definition, the zero frequency pseudo-WDF of the reconstructed wavefield $U(\mathbf{y}, t)$ is

$$
\begin{aligned}
W(\mathbf{y}, t)= & \int_{\left|t_{h}\right| \leq T} \mathrm{~d} t_{h} \int_{\left|\mathbf{y}_{h}\right| \leq Y} \mathrm{~d} \mathbf{y}_{h} U\left(\mathbf{y}-\frac{\mathbf{y}_{h}}{2}, t-\frac{t_{h}}{2}\right) \\
& \times U\left(\mathbf{y}+\frac{\mathbf{y}_{h}}{2}, t+\frac{t_{h}}{2}\right),
\end{aligned}
$$

where $Y$ and $T$ denote averaging windows in space and time, respectively. In general, $Y$ is three dimensional and $T$ is one dimensional. Then, the image $R_{\mathrm{IIC}}(\mathbf{y})$ is obtained by extracting the time $t=0$ from the pseudo-WDF, $W(\mathbf{y}, t)$, of the wavefield $U(\mathbf{y}, t)$ :

$R_{\mathrm{IIC}}(\mathbf{y})=W(\mathbf{y}, t=0)$.

The interferometric imaging condition represented by Eqs. 4 and 5 effectively reduces the artifacts caused by the random fluctuations in the wavefield by filtering out its rapidly varying components (Sava and Poliannikov 2008). In this paper, I use this imaging condition to attenuate noise caused by sparse data sampling or noise caused by random velocity variations. As suggested earlier, the interferometric imaging condition attenuates both types of noise at once, since it does not explicitly distinguish between the various causes of random fluctuations.

The parameters $Y$ and $T$ defining the local window of the pseudo-WDF are selected according to two criteria (Cohen 1995). First, the windows have to be large enough to enclose a representative portion of the wavefield which captures the random fluctuation of the wavefield. Second, the window has to be small enough to limit the possibility of cross-talk between various events present in the wavefield. Furthermore, cross-talk can be attenuated by selecting windows with different shapes, for example Gaussian or exponentially decaying. Therefore, we could in principle define the transformation in Eq. 4 more generally as

$$
\begin{aligned}
W(\mathbf{y}, t)= & \int_{\left|t_{h}\right| \leq T} \mathrm{~d} t_{h} W_{T}\left(t, t_{h}\right) \int_{\left|\mathbf{y}_{h}\right| \leq Y} \mathrm{~d} \mathbf{y}_{h} W_{Y}\left(\mathbf{y}, \mathbf{y}_{h}\right) \\
& \times U\left(\mathbf{y}-\frac{\mathbf{y}_{h}}{2}, t-\frac{t_{h}}{2}\right) U\left(\mathbf{y}+\frac{\mathbf{y}_{h}}{2}, t+\frac{t_{h}}{2}\right),
\end{aligned}
$$

where $W_{T}$ and $W_{Y}$ are weighting functions which could represent Gaussian, boxcar or any other local functions (Artman 2011, personal communication). For simplicity, in all examples presented in this paper, the space and time windows are rectangular with no tapering and the size is selected assuming that micro-earthquakes occur sufficiently sparse, i.e. the various sources are located at least twice as far in space and time relative to the wavenumber and frequency of the considered seismic event. Typical window sizes used here are 11 grid points in space and 5 grid points in time.

\section{Example}

I exemplify the interferometric imaging condition method with a synthetic example simulating the acquisition geometry of the passive seismic experiment performed at the San Andreas Fault Observatory at Depth (Chavarria et al. 2003; Vasconcelos et al. 2008). This numeric 


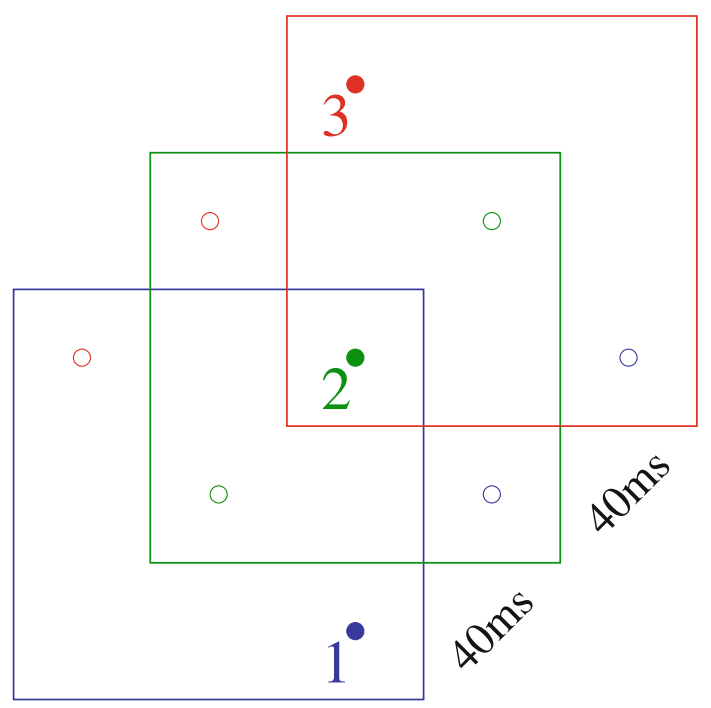

Fig. 3 Geometry of the sources used in the numeric experiment. The horizontal and vertical separation between sources is $250 \mathrm{~m}$. The sources are triggered with $40 \mathrm{~ms}$ delays in the order indicated by their numbers. Time $t=0$ is conventionally set to the triggering moment of source 2
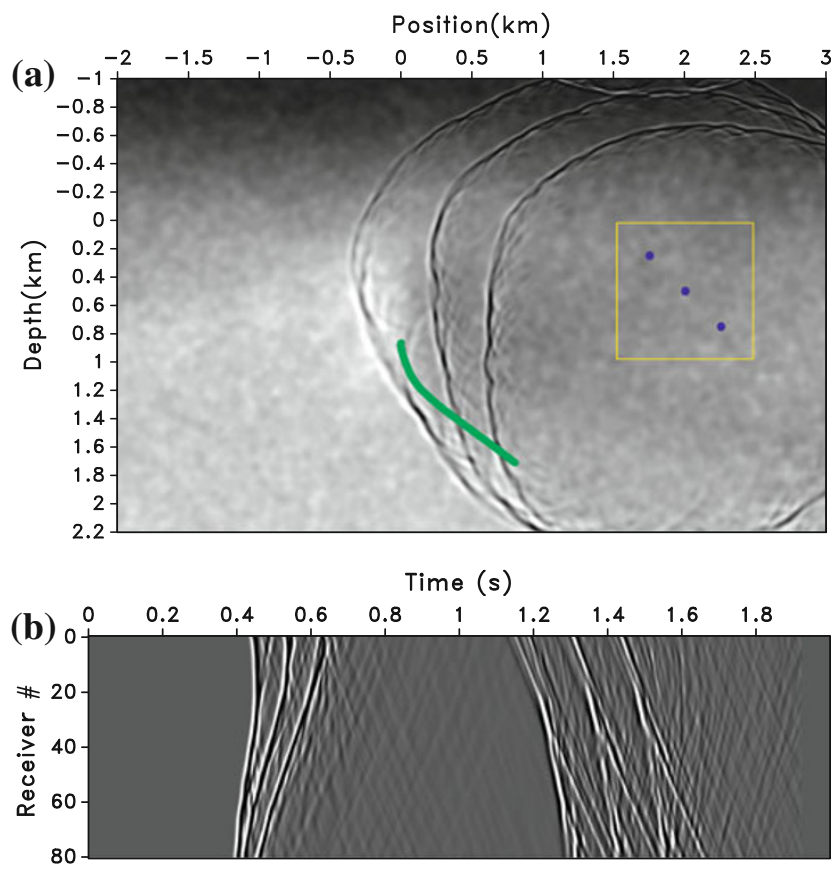

Fig. 4 a Wavefields simulated in random media and $\mathbf{b}$ data acquired with a dense receiver array. Overlain on the model and wavefield are the positions of the sources and borehole receivers. The boxed area corresponds to the images depicted in Fig. $6 a$ and $b$

experiment simulates waves propagating from three microearthquake sources located in the fault zone (Fig. 3), which are recorded in a deviated well located at a distance from the fault. For the imaging procedure described in this paper, the micro-earthquakes represent the seismic sources.
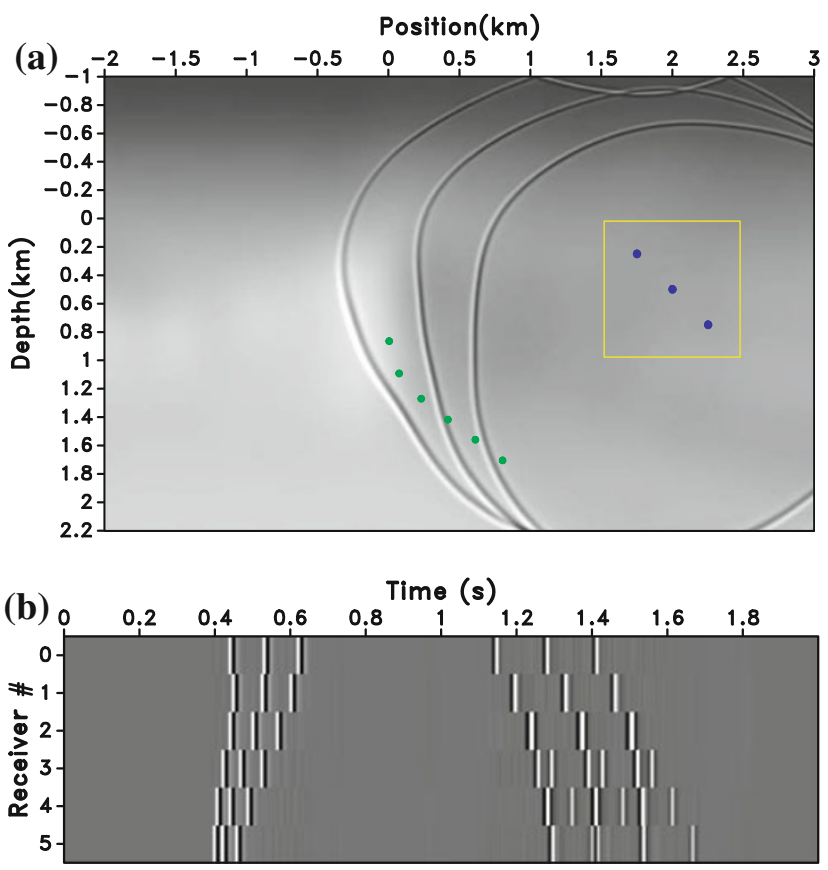

Fig. 5 a Wavefields simulated in smooth media and $\mathbf{b}$ data acquired with a sparse receiver array. Overlain on the model and wavefield are the positions of the sources and borehole receivers. The boxed area corresponds to the images depicted in Fig. $7 \mathrm{a}$ and $\mathrm{b}$

This experiment uses acoustic waves, corresponding to the situation in which we use the $\mathrm{P}$-wave mode recorded by the three-component receivers located in the borehole (Figs. 4b, 5b). The three sources are triggered $40 \mathrm{~ms}$ apart and the triggering time of the second source is conventionally taken to represent the origin of the time axis.

The goal of this experiment is to locate the source positions by focusing data recorded using dense acquisition in media with random fluctuations or by focusing data recorded using sparse acquisition arrays in media without random fluctuations. In the first case, the imaging artifacts are caused by the fact that data are imaged with a velocity model that does not incorporate all random fluctuations of the model used for data simulation, while in the second case, the imaging artifacts are caused by the fact that the data are sampled sparsely in the borehole array. The third case is a combination of acquisition with two sparse arrays, and imaging with an inaccurate velocity model.

Figures $6 \mathrm{a}$ and $\mathrm{b}, 7 \mathrm{a}$ and $\mathrm{b}$ and $8 \mathrm{a}$ and $\mathrm{b}$ show the wavefields reconstructed in reverse time around the target location. From left to right, the panels represent the wavefield at different times. As indicated earlier, the time at which source 2 focuses is selected as time $t=0$, although this convention is not relevant for the experiment and any other time could be selected as reference. The experiment depicted in Fig. 6a and $\mathrm{b}$ corresponds to modeling in a model with random fluctuations and 
Fig. 6 Images corresponding to migration of the densely sampled data (Fig. 4b) modeled in the random velocity by a conventional IC and b interferometric IC using the background velocity. The leftmost panel shows focusing at source 1 , the middle panel shows focusing at source 2 , and the right-most panel shows focusing at source 3 . The overlain dots represent the exact source positions (a)

$-40 \mathrm{~ms}$

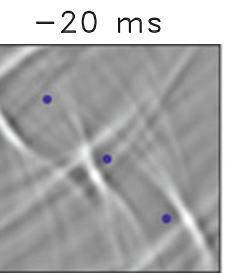

$0 \mathrm{~ms}$

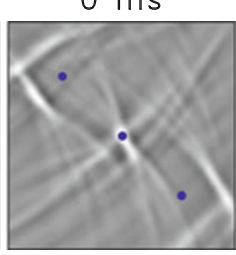

$20 \mathrm{~ms}$
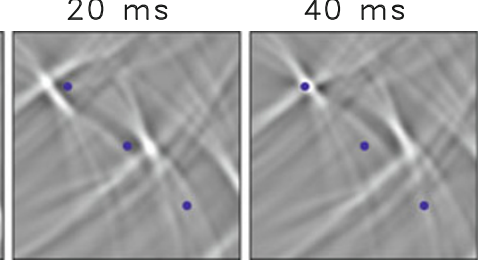

(b)

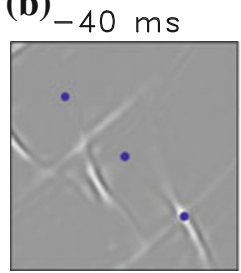
$-20 \mathrm{~ms}$ $0 \mathrm{~ms}$
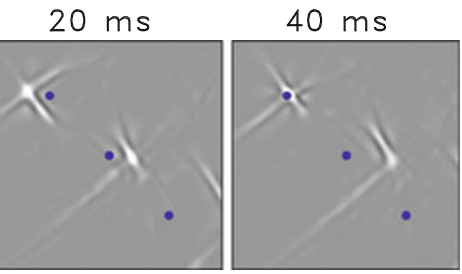

(a) $-40 \mathrm{~ms}$

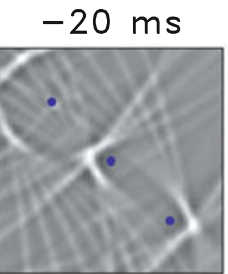

$0 \mathrm{~ms}$

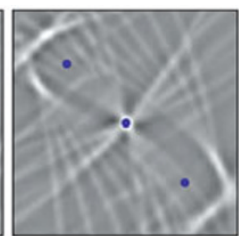

$20 \mathrm{~ms}$

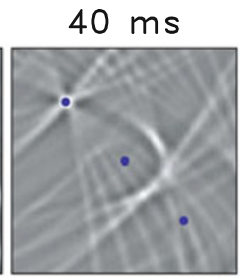

(b) $-40 \mathrm{~ms}$

$-20 \mathrm{~ms}$
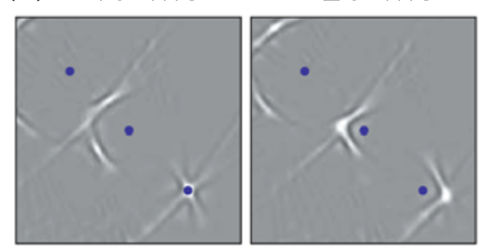

$0 \mathrm{~ms}$

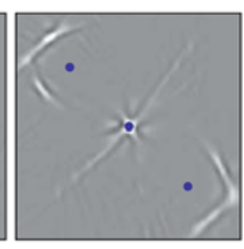

$20 \mathrm{~ms}$

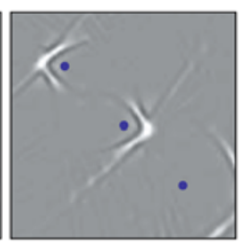

$40 \mathrm{~ms}$

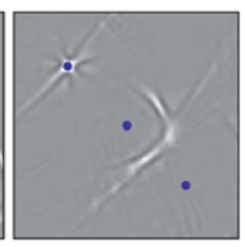

(a) $-40 \mathrm{~ms}$
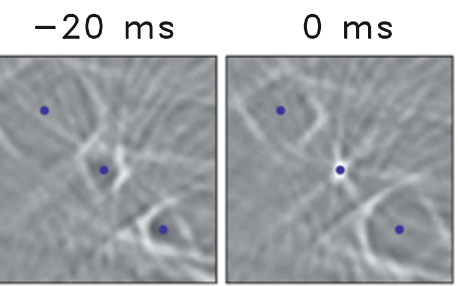

$20 \mathrm{~ms}$

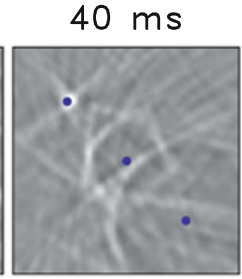

(b) $-40 \mathrm{~ms}$

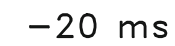

$0 \mathrm{~ms}$
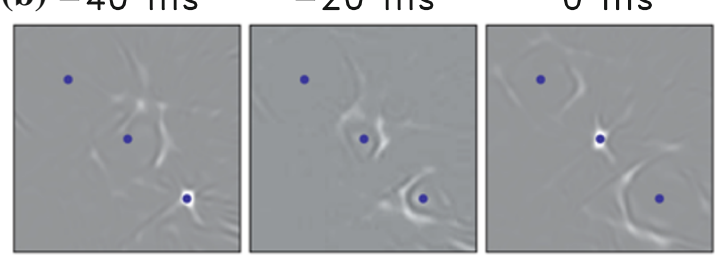

$20 \mathrm{~ms}$
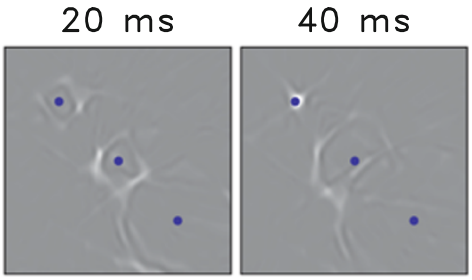

migration in a smooth background model. In this experiment, the data used for imaging are densely sampled in the borehole, i.e. there are 81 receivers separated by approximately $12 \mathrm{~m}$. In contrast, the experiment depicted in Fig. $7 \mathrm{a}$ and $\mathrm{b}$ corresponds to modeling and migration in the smooth background model. In this experiment, the data are sparsely sampled in the borehole, i.e. there are only six receivers obtained by selecting every 16 th receiver from the original set. In all cases, panels (a) correspond to imaging with a conventional imaging condition, i.e. simply 

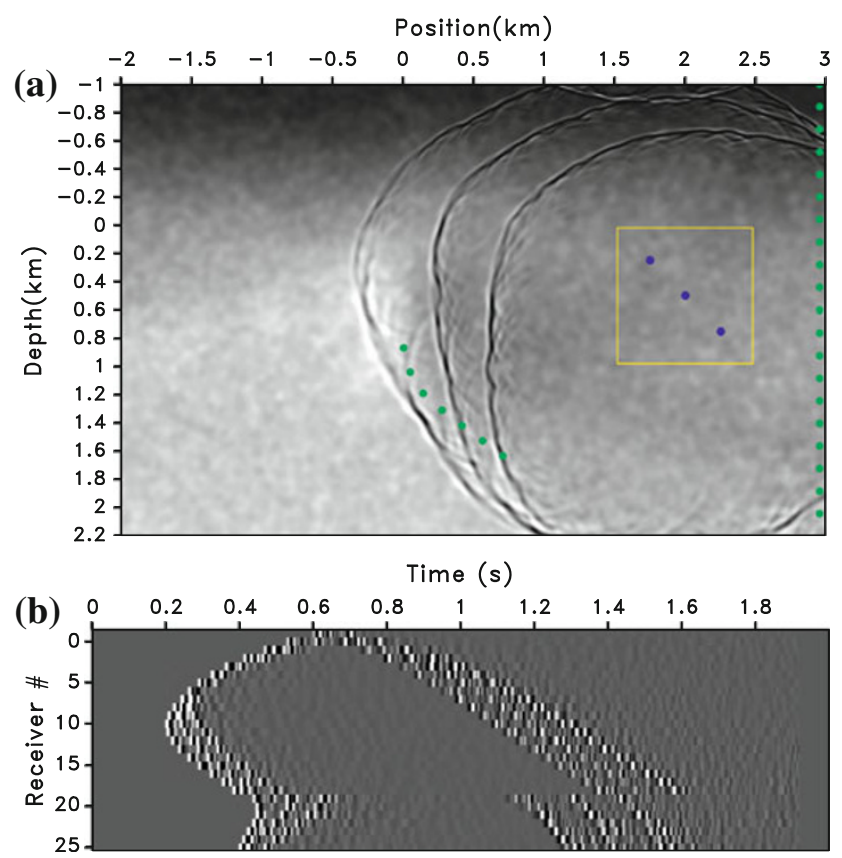

Fig. 9 a Wavefields simulated in random media and $\mathbf{b}$ data acquired with two sparse receiver arrays. Overlain on the model and wavefield are the positions of the sources and borehole receivers. The boxed area corresponds to the images depicted in Fig. $8 \mathrm{a}$ and b. The top traces in $\mathbf{b}$ correspond to the vertical array, and the other traces correspond to the sparse deviated array

select the reconstructed wavefield at various times, and panels (b) correspond to imaging with the interferometric imaging condition, i.e. select various times from the wavefield transformed with a pseudo-WDF of 11 grid points in space and 5 grid points in time. For this example, WDF window corresponds to $44 \mathrm{~m}$ in space and $2 \mathrm{~ms}$ in time.

Figure 6a shows significant random fluctuations caused by wavefield reconstruction using an inaccurate velocity model. The fluctuations caused by the random velocity and encoded in the recorded data are not corrected during wavefield reconstruction and they remain present in the model. Likewise, Fig. 7a shows significant random fluctuations caused by reconstruction using the sparse borehole data. However, the pseudo-WDF applied to the reconstructed wavefields attenuates the rapid wavefield fluctuations and leads to sparser, better focused images that are easier to use for source location. This conclusion applies equally well for the experiments depicted in Fig. 6a and b or $7 \mathrm{a}$ and $\mathrm{b}$.

The final example corresponds to the case of acquisition with two separate sparse arrays (Fig. 9a, b). As expected, the wavefields are far less noisy after the application of the $\mathrm{WDF}$, and the focusing is increased due to the larger array aperture. This facilitates an automatic procedure for focusing identification, since most of the spurious noisy is eliminated from the image.
Finally, I note that the 2D imaging results from this example show better focusing than what would be expected in 3D. This is simply because the $1 \mathrm{D}$ acquisition in the borehole cannot constrain the 3D location of the microearthquakes, i.e. the azimuthal resolution is poor, especially if scatterers are not present in the model used for imaging. This situation can be improved using data acquired in several boreholes or using additional information extracted from the wavefields, e.g. polarization of multicomponent data.

\section{Conclusions}

The interferometric imaging condition used in conjunction with time-reverse imaging reduces the artifacts caused by random velocity fluctuations that are unaccounted-for in imaging and by the sparse wavefield sampling on the acquisition array. The images produced by this procedure are crisper and support automatic picking of micro-earthquake locations. Imaging with sparse arrays allows increased aperture for identical acquisition cost with that of a narrower but denser array. At the same time, a larger aperture improves focusing of the events, thus facilitating automatic event identification. The interferometric imaging procedure has a similar structure to conventional imaging and the moderate cost increase is proportional to the size of the windows used by the pseudo-Wigner distribution functions. The source positions obtained using this procedure can be used to monitor fluid injection or for studies of naturally occurring earthquakes in fault zones.

Acknowledgments This work is supported by the sponsors of the Center for Wave Phenomena at Colorado School of Mines and by a research grant from ExxonMobil. The reproducible numeric examples in this paper use the Madagascar open-source software package freely available from http://www.reproducibility.org.

Open Access This article is distributed under the terms of the Creative Commons Attribution Noncommercial License which permits any noncommercial use, distribution, and reproduction in any medium, provided the original author(s) and source are credited.

\section{References}

Artman B, Podladtchikov I, Witten B (2010) Source location using time-reverse imaging. Geophys Prospect 58:861-873

Borcea L, Papanicolaou G, Tsogka C (2006) Coherent interferometric imaging in clutter. Geopysics 71:SI165-SI175

Chavarria J, Malin P, Shalev E, Catchings R (2003) A look inside the San Andreas Fault at Parkfield through vertical seismic profiling. Sci Agric 302:1746-1748

Cohen L (1995) Time frequency analysis: Signal processing series. Prentice Hall, Englewood Cliffs 
Gajewski D, Anikiev D, Kashtan B, Tessmer E, Vanelle C (2007) Localization of seismic events by diffraction stacking, In: 76th annual international meeting, SEG, Expanded Abstracts, pp 1287-1291

Gajewski D, Tessmer E (2005) Reverse modelling for seismic event characterization. Geophys J Int 163:276-284

Rentsch S, Buske S, Luth S, Shapiro SA (2004) Location of seismicity using Gaussian beam type migration. In: 74th annual international Meeting. Society of Exploration Geophysicists, pp 354-357

Rentsch S, Buske S, Luth S, Shapiro SA (2007) Fast location of seismicity: a migration-type approach with application to hydraulic-fracturing data. Geophysics 72:S33-S40
Sava P, Poliannikov O (2008) Interferometric imaging condition for wave-equation migration. Geophysics 73:S47-S61

Vasconcelos I, Snieder R, Sava P, Taylor T, Malin P, Chavarria A (2008) Drill bit noise illuminates the San Andreas fault. EOS. Trans Am Geophys Union 89:349

Waldhauser F, Ellsworth W (2000) A double-difference earthquake location algorithm: method and application to the northern Hayward fault. Bull Seismol Soc Am 90:1353-1368

Wigner E (1932) On the quantum correction for thermodynamic equilibrium. Phys Rev 40:749-759 